\title{
INHIBICIÓN Y MEMORIA DE TRABAJO: MARCADORES DIFERENCIALES DE LAS DIFICULTADES EN CÁLCULO Y RESOLUCIÓN DE PROBLEMAS EN EDUCACIÓN INFANTIL
}

\author{
Jessica Mercader Ruiz \\ Universitat Jaume I \\ mercader@uji.es \\ Aïda Puig Lleixà \\ Universitat Jaume I \\ al313206@uji.es \\ Rosa M. Rodrigo Carrión \\ Universitat Jaume I \\ al313661@uji.es \\ Laura Abellán Roselló \\ Universidad Internacional de la Rioja \\ laura.abellan@unir.net \\ Rebeca Siegenthaler Hierro \\ Universitat Jaume I \\ siegenth@uji.es
}

Fecha de Recepción: 16 Mayo 2019

Fecha de Admisión: 25 Septiembre 2019

\section{RESUMEN}

El objetivo del presente estudio consistió en determinar si las funciones ejecutivas de memoria de trabajo (MT) e inhibición se constituyen como marcadores diferenciales de las dificultades en los dominios de cálculo y resolución de problemas al inicio de la escolaridad. La muestra estuvo compuesta por 208 preescolares de 5 a 6 años (edad media $=70.17$ meses; DT $=3.51 ; 47.6 \%$ niñas y $52.4 \%$ niños). Se administraron distintas tareas neuropsicológicas de funcionamiento ejecutivo para evaluar la inhibición (visual y auditiva) y la MT (verbal y viso-espacial). Los participantes se categorizaron en 4 grupos en función de su rendimiento en los subtest de cálculo y resolución de problemas de la batería TEDI-MATH (Grégoire, Noël, y Van Nieuwenhoven, 2005): dificultades iniciales en cálculo (DC, $n=17$ ); dificultades iniciales en resolución de problemas (DRP, $n=13$ ); dificultades iniciales en ambos dominios $(D C+D R P=20)$ y rendimiento medio $(R M, n=158)$. Los resultados revelaron un peor funcionamiento del grupo con DC+DRP en todas las tareas de inhibición y MT verbal en comparación con el grupo con RM. Se destaca una mayor afectación del grupo con DC en inhibición visual, y del grupo con DRP en MT verbal en comparación con los niños sin problemas. La tarea de inhibición auditiva es la única capaz de discriminar entre niños con DC+DRP y niños con dificultades en único dominio. No se encontraron dife- 


\title{
INHIBICIÓN Y MEMORIA DE TRABAJO: MARCADORES DIFERENCIALES DE LAS DIFICULTADES EN CÁLCULO Y RESOLUCIÓN DE PROBLEMAS EN EDUCACIÓN INFANTIL
}

rencias en las tareas de MT viso-espacial en ninguno de los casos. Se comentan las implicaciones de estos hallazgos para la investigación y la práctica psicoeducativa.

Palabras clave: memoria de trabajo; inhibición; cálculo; resolución de problemas; Educación Infantil

\begin{abstract}
Inhibition and working memory: differential markers of arithmetic and solving problems difficulties in Kindergarten. The aim of this study was to determine if the executive functions of working memory (WM) and inhibition are constituted as differential markers of arithmetic and problem solving difficulties at schooling beginning. The sample consisted of 208 kindergarteners aged 5 to 6 years (mean age $=70.17$ months; $\mathrm{SD}=3.51 ; 47.6 \%$ girls and $52.4 \%$ boys). Different executive functioning neuropsychological tasks were administered to assess visual and auditory inhibition, and verbal and viso-spatial WM. Participants were categorized into 4 groups based on their performance in the arithmetic and problem solving subtests of TEDI-MATH (Grégoire, Noël, and Van Nieuwenhoven, 2005): initial arithmetic difficulties (AD, $n=17$ ) ; initial problem solving difficulties (PSD, $n=13$ ); initial difficulties in both domains ( $A D+P S D, n=20$ ) and average performance (AP, $n$ $=158$ ). Results revealed a worse functioning of $A D+P S D$ group in all tasks of inhibition and verbal WM compared to the AP group. Analysis also showed greater impairment of the AD group in visual inhibition and the PSD group in verbal WM in comparison with children without math problems. The task of auditory inhibition is the only one able to discriminate between DC + DRP group and children with difficulties in a single domain. No differences were found in the viso-spatial WM tasks. The implications of these findings for research and psychoeducational practice are discussed.
\end{abstract}

Keywords: working memory; inhibition; arithmetic; problem solving; Kindergarten

\section{INTRODUCCIÓN}

La competencia matemática se va construyendo desde los primeros años de vida, debido a que las capacidades matemáticas van evolucionando hacia una mayor complejidad a medida que avanza el desarrollo cognitivo (Alsina, 2012). Concretamente el cálculo y la resolución de problemas son habilidades que tienen como finalidad llevar a contextos cotidianos los conceptos matemáticos y, además, constituyen, para algunos autores, el centro del pensamiento matemático formal (Jordan, Glutting y Ramineni, 2010). Aunque es en las primeras etapas donde estas competencias matemáticas se deben desarrollar y adquirir de la manera más efectiva posible (Alsina, 2012), según cómo se adquieran se determinará los procesos posteriores. En muchas ocasiones, las dificultades iniciales en cálculo y resolución de problemas son un signo de alerta para la detección de dificultades específicas en matemáticas que no se desarrollaran hasta después de varios años de escolaridad (Desoete, Roeyers y De Clerq, 2004). Por todo ello, es importante identificar lo antes posible qué factores asocian al rendimiento en cálculo y resolución de problemas en etapas tempranas, para poder prevenir dificultades posteriores (Passolunghi y Lanfranchi, 2012).

Durante las etapas iniciales del desarrollo se ha ratificado que el funcionamiento ejecutivo (FE) está estrechamente relacionado con el rendimiento en cálculo (Bull y Lee, 2014) y en resolución de problemas matemáticos (Bull y Scerif, 2001). Concretamente, se destacan las investigaciones centradas en las relaciones entre FE de memoria de trabajo (MT) e inhibición, dado su carácter central y temprano en el desarrollo del FE (Diamond, 2013).

En lo relativo al cálculo, diferentes estudios muestran que los distintos componentes de la MT (verbal y visoespacial) son fundamentales para la resolución de operaciones aritméticas simples (Peng, Barnes, Namkug y Sun, 2016), siendo especialmente relevante el componente viso-espacial para el cálculo en muestras de alumnado en las primeras etapas educativas (McKenzie et al., 2003). Por otra parte, aunque la relación entre la inhibición y el cálculo ha sido menos estudiada, distintos trabajos resaltan la importancia de la inhibición para un desarrollo matemático adecuado (Blair y Razza, 2007; Bull, Espy, y Wiebe, 2008). En esta línea algunos estudios han demostrado que los sujetos con habilidades aritméticas inferiores muestran dificultades en tareas de inhibición (Van der 
Sluis et al., 2004; Wang, Tasi y Yang, 2012), y de MT (Szucs, Devine, Soltesz, Nobes y Gabriel, 2013), especialmente en tareas que tienen que ver con información numérica y gráfica (Peng y Fuchs, 2016).

Respecto a resolución de problemas matemáticos, la MT permite almacenar temporalmente y manipular información relevante del problema, lo que posibilita ejecutar procedimientos aritméticos complejos. Algunos estudios concluyen que la MT (especialmente, el componente verbal) es un importante predictor de la resolución de problemas en Educación Primaria (Anderson, 2010; Lee, Bull, Pe y Ho, 2011) y en Educación Infantil (Bull, Espy y Wiebe, 2008; Holmens y Adams, 2006). En lo que se refiere a la inhibición, contribuye a la supresión de las respuestas dominantes, como completar las operaciones por el orden en que se presentan en el problema, en lugar de por el orden en el que se debe resolver para llegar a la respuesta adecuada. Así, diferentes trabajos revelan que la inhibición es una variable contribuye a la capacidad de los niños para resolver problemas de operaciones simples de un paso y de múltiples pasos en distintas etapas educativas (Agostino, Johnson, y PascualLeone, 2010; Blair y Razza, 2007; Espy, et al., 2004). Finalmente, se han encontrado que el alumnado con dificultades en resolución de problemas, puede tener afectación en los procesos de MT (Andersson, 2010; Andersson y Lyxel, 2007) e inhibición (Passolunghi y Lanfranchi, 2012).

En resumen, a pesar de la amplia investigación que muestra la asociación entre las FE de MT e inhibición con el desarrollo matemático y sus dificultades en las etapas superiores, existen escasos estudios que profundicen en el conocimiento de dicha asociación en los primeros años de la escolaridad, siendo sus resultados menos concluyentes. Igualmente, son pocos los estudios que han incluido sujetos con dificultades en ambos dominios de forma diferenciada, así como medidas neuropsicológicas de inhibición y MT. Por ello, son necesarios más trabajos que analicen estas diferencias en variables desde una preventiva, que abarque la etapa de Educación Infantil y contemple las dificultades en los distintos procesos relacionados con el cálculo y la resolución de problemas matemáticos.

\section{OBJETIVOS DE LA INVESTIGACIÓN}

El objetivo del presente estudio consistió en determinar si las funciones ejecutivas de memoria de trabajo (MT) e inhibición se constituyen como marcadores diferenciales de las dificultades en los dominios de cálculo y resolución de problemas al inicio de la escolaridad.

\section{MUESTRA Y/O PARTICIPANTES}

La muestra estuvo conformada por 208 sujetos escolarizados en 15 centros de las provincias de Castellón y Valencia. Se seleccionaron 6 participantes por aula de Educación Infantil de 5 y 6 años (47.6\% niñas y 52.4\% niños; edad media $=70.17$ meses; DT = 3.51) mediante muestreo aleatorio simple. El $63.5 \%$ asistían a centros públicos y el $36.6 \%$ a concertados. El 88 \% tenían nacionalidad española. Se calculó el Cl equivalente de los participantes siguiendo las directrices de Spreen, Sherman y Straus (2006), a través de las subpruebas de vocabulario y cuadrados del WIPPSI (Weschler, 1967). La media de CI fue de 98.72 (DT = 12.19; rango 70 - 126). Todos los participantes tenían un $\mathrm{Cl}$ equivalente superior a 70 y no presentaban deficiencias sensoriales graves, anomalías neurobiológicas, trastornos psicológicos o depravación socio-cultural.

La muestra se dividió en función de las puntuaciones percentiles obtenidas en los subtest de operaciones aritméticas con enunciado aritmético (cálculo) y operaciones aritméticas con enunciado (resolución de problemas) verbal del test TEDI-MATH (Grégoire, Noël, y Van Neiuwenhoven, 2005). El grupo con dificultades iniciales en cálculo (DC) estuvo conformado por los sujetos con una puntuación igual o inferior al percentil 25 en la tarea de cálculo, pero con un nivel adecuado en la tarea de resolución de problemas $(n=17)$. Los participantes cuyas puntuaciones fueron iguales 0 inferiores al percentil 25 en la tarea de resolución de problemas, pero sin dificultades iniciales en la tarea de cálculo, formaron parte del grupo DRP $(n=13)$. El grupo DC+DRP estuvo compuesto por niños y niñas con puntuaciones iguales 0 inferiores al percentil 25 en ambas subpruebas $(n=20)$. Aquellos sujetos que obtuvieron una puntuación superior al percentil 25 en ambos subtest conformaron el grupo de RM 


\section{INHIBICIÓN Y MEMORIA DE TRABAJO: MARCADORES DIFERENCIALES DE LAS DIFICULTADES EN CÁLCULO Y RESOLUCIÓN DE PROBLEMAS EN EDUCACIÓN INFANTIL}

( $n=158)$. Se encontraron diferencias estadísticamente significativas entre los grupos en la variable $\mathrm{Cl}$ equivalente $\left[\mathrm{F}_{3,204}=9.86, p<.001,{ }^{2} p=.127\right]$ que fueron tenidas en cuenta en los posteriores análisis (véase Tabla 1).

Tabla 1. Criterios para el establecimiento de los grupos según la puntuación en los subtest seleccionados de la batería TEDI-MATH (Grégoire et al., 2005) y medias y DT de Cl Equivalente.

\begin{tabular}{|c|c|c|c|c|c|}
\hline & \multirow{2}{*}{$\begin{array}{l}\text { Operaciones } \\
\text { aritméticas con } \\
\text { enunciado } \\
\text { aritmético } \\
\text { (Cálculo) }\end{array}$} & \multirow{2}{*}{$\begin{array}{l}\text { Operaciones } \\
\text { aritméticas con } \\
\text { enunciado verbal } \\
\text { (Resolución de } \\
\text { problemas) }\end{array}$} & \multirow{2}{*}{$\mathrm{N}$} & \multicolumn{2}{|c|}{$\mathrm{CI}$ equivalente } \\
\hline & & & & M & DT \\
\hline $\begin{array}{l}\text { Dificultades } \\
\text { iniciales en } \\
\text { cálculo (DC) }\end{array}$ & $\mathrm{PC} \leq 25$ & $\mathrm{PC} \geq 25$ & 17 & 94.71 & 12.81 \\
\hline $\begin{array}{l}\text { Dificultades } \\
\text { iniciales en } \\
\text { resolución de } \\
\text { problemas } \\
\text { (DRP) }\end{array}$ & $\mathrm{PC} \geq 25$ & $\mathrm{PC} \leq 25$ & 13 & 88.23 & 11.84 \\
\hline $\begin{array}{c}\text { Dificultades } \\
\text { iniciales en ambos } \\
\text { dominios } \\
\text { (DC+DRP) }\end{array}$ & $\mathrm{PC} \leq 25$ & $\mathrm{PC} \leq 25$ & 20 & 90.55 & 8.99 \\
\hline $\begin{array}{l}\text { Rendimiento } \\
\text { medio (RM) }\end{array}$ & $\mathrm{PC} \geq 25$ & $\mathrm{PC} \geq 25$ & 158 & 101.05 & 11.57 \\
\hline
\end{tabular}

\section{METODOLOGÍA Y/O INSTRUMENTOS UTILIZADOS}

\section{Instrumentos}

\section{Evaluación neuropsicológica del FE}

Inhibición. Para evaluar la inhibición se administraron las tareas de Stroop Sol-Luna (Archibald y Kerns, 1999) con estímulos visuales y el test de Golpeteo (Luria, 1966) con estímulos auditivos. La primera tarea, consta de dos condiciones distintas, en las que el niño debe observar 30 imágenes de soles y lunas distribuidas aleatoriamente en filas. En la evaluación congruente, deben nombrar tan rápido como puedan (durante 45") "sol" 0 "luna" en las imágenes con dicha representación. En la incongruente, deben responder "sol" cuando aparece la figura de una luna y "luna" cuando sea un sol. La fiabilidad test-retest para la condición incongruente es de .91 (Archibald y Kerns, 1999). La segunda tarea, también presenta dos condiciones con 12 ensayos cada una. Primero el niño debe imitar al evaluador dando el mismo número de golpes sobre un soporte $\left(\begin{array}{lll}1 & 0 & 2\end{array}\right)$. Seguidamente, debe realizar el número de golpes contrario al que realiza el examinador. Se ha verificado la fiabilidad de la tarea en .87 (Diamond y Taylor, 1996).

Memoria de trabajo viso-espacial. Para evaluar el componente viso-espacial de la MT se utilizaron la prueba de Odd-One-Out (Henry y MacLean, 2003) y el test de Memoria de Laberintos (Pickering, Baqués, y Gathercole, 1999). La primera, consta de 4 niveles con 4 pruebas cada uno. Se presentan láminas con filas de 3 figuras en cada ensayo. El participante debe señalar la figura distinta y recordar su posición (izquierda, centro, derecha) al finalizarlo. En la segunda tarea, se muestran 12 laberintos con las rutas marcadas, el niño debe representar la misma ruta en un laberinto en blanco (hay 3 niveles de dificultad). La fiabilidad es de .81 para ambas tareas (Alloway, Gathercole, y Pickering, 2006). 
Memoria de trabajo verbal. Para evaluar la MT verbal se aplicaron la tarea de Dígitos Inversos (Pickering et al.,1999) y el test de Conteo (Siegel y Ryan, 1989). En la primera, se muestran series, con 4 ensayos cada una, de 2 a 9 dígitos. Los niños deben decir las series de forma inversa al examinador. En la segunda, se presentan puntos azules y amarillos distribuidos aleatoriamente en una carta. Los niños deben decir cuántos puntos azules aparecen y recordar las cantidades al final del ensayo. Consta de 3 niveles con 4 ensayos (2 a 4 cartas). La fiabilidad test-retest es de .64 (Alloway et al., 2006) y .62 (Gathercole, Pickering, Ambridge, y Wearing, 2004) respectivamente. Para los análisis se utilizaron las puntuaciones directas de ensayos correctos en todos casos.

Evaluación de la competencia inicial en cálculo y resolución de problemas. TEDI-MATH (Grégoire et al., 2005). Se seleccionaron las subpruebas de operaciones con enunciado aritmético y operaciones con enunciado verbal para evaluar las habilidades matemáticas de cálculo y resolución de problemas. En la primera, se presentan 5 láminas de sumas simples. En la segunda, 17 problemas escritos. El sujeto debe resolverlos mentalmente (releyendo si es necesario) y decir la respuesta. La fiabilidad test-retest es de 0.71 y 0.75 respectivamente (Grégoire et al., 2005). Se establecieron los grupos en base a la puntuación percentil obtenida en cada una de las tareas.

\section{Procedimiento}

La investigación se autorizó por el Comité ético de la Universidad Jaume I y la Conselleria d' Educació, Cultura i Esport de la Comunidad Valenciana. Asimismo, se obtuvo el consentimiento de los centros escolares y permiso de los padres. Se utilizaron las aulas habilitadas con condiciones óptimas de iluminación, ventilación y aislamiento. La evaluación fue llevada a cabo por evaluadores experimentados en 2 sesiones individualizadas de evaluación (30) con cada niño.

\section{Análisis estadísticos}

Para la realización de los análisis estadísticos, se empleó el programa Statistical Package for the Social Science, versión 22.00 (SPSS Inc., Chicago IIlinois, USA). Para comprobar las diferencias en las variables de FE evaluadas mediante tareas neuropsicológicas de inhibición y MT verbal y viso-espacial entre los distintos grupos generados en función del rendimiento inicial en cálculo y resolución lector (DC, DRP, DC+DRP y RM), se llevó a cabo un análisis multivariado de la covarianza (MANCOVA), introduciendo el $\mathrm{Cl}$ equivalente como variable de control. Se comprobaron los efectos principales $(p<.05)$, se realizaron comparaciones post-hoc por el método Bonferroni y se calculó el valor de $2 p$ para comprobar la fuerza de la asociación.

\section{RESULTADOS ALCANZADOS}

En la Tabla 2 se recogen los análisis referidos a las diferencias en las tareas neuropsicológicas de FE. El efecto principal de grupo resultó estadísticamente significativo [Wilks' Lambda ()$=.70, \mathrm{~F}_{18}, 560=4.17, p<.001$, $2 p=.112]$. Los ANCOVA's de confirmación mostraron diferencias estadísticamente significativas en las tareas de inhibición (Stroop Sol Luna, $\mathrm{F}_{3,203}=7.31, p<.001,{ }^{2} p=.098$; Test de Golpeteo, $\mathrm{F}_{3,203}=17.20, p<.001$, ${ }^{2} p=.203$ ) y MT verbal (Dígitos Inversos, $\mathrm{F}_{3,203}=17.20, p<.001,{ }^{2} p=.050$; Test de Conteo, $\mathrm{F}_{3,203}=8.76$, $\left.p<.001,{ }^{2} p=.115\right)$. No se encontraron diferencias estadísticamente significativas en las tareas de MT viso-espacial en ninguno de los casos (Odd-One-Out, $\mathrm{F}_{3}, 203=2.19, p>.05,2 p=.031$; Laberintos, $\mathrm{F}_{3,203}=.065, p>$ $.05,2 p=.001)$

Las comparaciones por pares a posteriori (Bonferroni) revelaron diferencias estadísticamente significativas entre el grupo con DC y el grupo con RM en la tarea de inhibición Stroop Sol-Luna $(p=.010)$. El grupo con DRP presentó diferencias estadísticamente significativas con el grupo con RM en la tarea de MT verbal de Conteo ( $p$ $=.050)$. El grupo con dificultades en ambos dominios (DC+DRP) mostró diferencias con el grupo con RM en todas las tareas en las que Ios ANCOVA mostraron diferencias significativas (Stroop Sol-Luna, $p=.001$; Test de Golpeteo, $p<.001$; Dígitos Inversos, $p=.022$; Test de Conteo, $p<.001$ ). Se encontraron también diferencias entre el grupo DC+DRP en el test de inhibición de Golpeteo con el resto de grupos con dificultades ( $p \leq .001)$, así como con el grupo con DC en la tarea de MT verbal de Conteo $(p=.017)$. 


\section{INHIBICIÓN Y MEMORIA DE TRABAJO: MARCADORES DIFERENCIALES DE LAS DIFICULTADES EN CÁLCULO Y RESOLUCIÓN DE PROBLEMAS EN EDUCACIÓN INFANTIL}

Tabla 2. Comparaciones entre los grupos con y sin dificultades iniciales en cálculo y resolución de problemas en las tareas neuropsicológicas de FE.

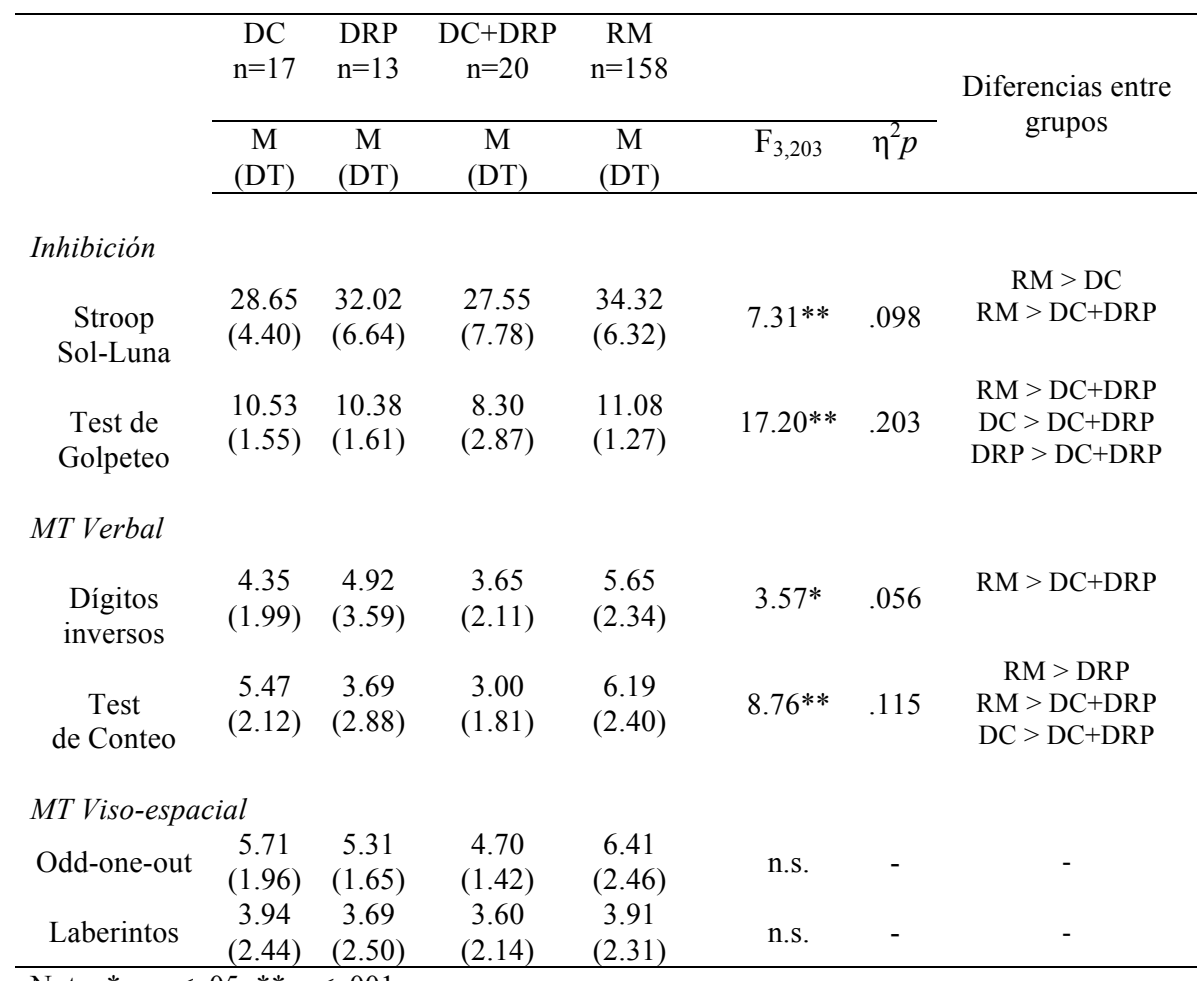

Nota: $*=p<.05 ; * * p<.001$

$\mathrm{DC}=$ Dificultades iniciales en cálculo; DRP $=$ Dificultades iniciales en resolución de problemas; $\mathrm{DC}+\mathrm{DRP}=$ Dificultades iniciales en ambos dominios; $\mathrm{RM}=$ Rendimiento medio $\mathrm{MT}=$ Memoria de trabajo

\section{DISCUSIÓN}

Esta investigación se propuso como objetivo examinar las diferencias en distintas tareas neuropsicológicas de inhibición y MT entre alumnado con dificultades iniciales en cálculo, resolución de problemas, déficits en ambos dominios y estudiantes con un rendimiento medio en Educación Infantil.

En la línea de trabajos anteriores realizados en distintas etapas del desarrollo, las tendencias de las medias muestran que los estudiantes con dificultades matemáticas poseen un peor rendimiento en FE en comparación con los estudiantes con RM (Bull y Lee, 2014; Bull y Scerif, 2001). Ahora bien, los resultados muestran una afectación diferencial de las funciones ejecutivas analizadas (MT e inhibición) según el dominio matemático en el que se presentan las dificultades.

En relación al cálculo, en contra de estudios anteriores que destacan el papel fundamental de la MT y especialmente del componente viso-espacial en las primeras etapas educativas (McKenzie et al., 2003; Peng et al., 2016), Ios resultados obtenidos en el presente trabajo muestran que es la tarea de inhibición visual la que diferencia a niños con dificultades iniciales en este dominio y niños sin problemas. Estos resultados están en la línea 
de estudios que destacan la importancia de la inhibición (Van der Sluis et al., 2004; Wang et al., 2012), especialmente aquella que tiene que ver con el control de la interferencia cuando se maneja información gráfica (Peng y Fuchs, 2016).

En lo que se refiere a la resolución de problemas, los resultados muestran una estrecha relación entre las dificultades iniciales en resolución de problemas y el componente de MT verbal (Bull et al., 2008; Holmens y Adams, 2010). Mucho menores son los resultados obtenidos en relación a la inhibición.

Como cabía esperar, el grupo con RM obtiene diferencias destacadas con el grupo con dificultades en ambos dominios, tanto en inhibición visual y auditiva (Passolunghi y Lanfranchi, 2012; Peng y Fuchs, 2016), como en MT verbal (Anderson y Lyxell, 2007; Peng et al., 2016). Además, cabe destacar que la tarea de inhibición auditiva ha sido la única capaz de diferenciar entre niños con dificultades en un solo dominio (cálculo o resolución de problemas) y estudiantes con la condición comórbida.

Finalmente, no se han encontrado diferencias en las tareas de MT viso-espacial en ninguno de los casos, en contraposición a otros trabajos (McKenzie et al., 2003). Este hecho podría explicarse en base a factores como la inclusión de grupos diferenciados en función del dominio matemático afectado, la edad de la muestra o las pruebas utilizadas. Futuros trabajos deberían profundizar en esta cuestión e incorporar más variables de FE que han demostrado su influencia en el rendimiento en cálculo y resolución de problemas, como por ejemplo la flexibilidad (Bull y Lee, 2014). También deberían tenerse en cuenta factores como la motivación y el estilo atribucional de cada individuo y factores socioemocionales que han demostrado tener relación con las dificultades matemáticas (Mercader, Presentación, Siegenthaler, Molinero y Miranda, 2017).

\section{CONCLUSIONES}

El presente trabajo sugiere que el FE temprano posee un papel relevante en el rendimiento del cálculo y resolución de problemas desde etapas iniciales de escolaridad. Por lo que podría concluirse que el currículo debería fundamentarse en la investigación rigurosa y de calidad, centrándose en competencias tempranas como el FE y especialmente en la inhibición y MT (verbal), que fomenten una evolución positiva en el rendimiento en el área de matemáticas y concretamente en el cálculo y la resolución de problemas.

\section{REFERENCIAS BIBLIOGRÁFICAS}

Agostino, A., Johnson, J., y Pascual-Leone, J. (2010). Executive functions underlying multiplicative reasoning: Problem type matters. Journal of Experimental Child Psychology, 105(4), 286-305.

Alloway, T. P., Gathercole, S. E., y Pickering, S. J. (2006). Verbal and visuospatial short term and working memory in children: Are they separable? Child Development, 77(6), 1698-1716. doi: 10.1111/j.14678624.2006.00968.x

Alsina, Á. (2012). Más allá de los contenidos, los procesos matemáticos en Educación Infantil. Edma 0-6: Educación Matemática en la infancia (1), 1-14.

Andersson, U. (2010). Skill development in different components of arithmetic and basic cognitive functions: Findings from a 3-year longitudinal study of children with different types of learning difficulties. Journal of Educational Psychology, 102(1), 115.

Andersson, U., y Lyxell, B. (2007). Working memory deficit in children with mathematical difficulties: A general or specific deficit? Journal of Experimental Child Psychology, 96(3), 197-228.

Archibald, S. J., y Kerns, K. A. (1999). Identification and description of new tests of executive functioning in children. Child Neuropsychology, 5(2), 115-129. doi: 10.1076/chin.5.2.115.3167

Blair, C., y Razza, R. P. (2007). Relating effortful control, executive function, and false belief understanding to emerging math and literacy ability in kindergarten. Child Development, 78(2), 647-663. doi: 10.1111/j.14678624.2007.01019.x

Bull, R., Espy, K. A., y Wiebe, S. A. (2008). Short-term memory, working memory, and executive functioning in preschoolers: Longitudinal predictors of mathematical achievement at age 7 years. Developmental 


\section{INHIBICIÓN Y MEMORIA DE TRABAJO: MARCADORES DIFERENCIALES DE LAS DIFICULTADES EN CÁLCULO Y RESOLUCIÓN DE PROBLEMAS EN EDUCACIÓN INFANTIL}

Neuropsychology, 33(3), 205-228.

Bull, R., y Lee, K. (2014). Executive functioning and mathematics achievement. Child Development Perspectives, 8(1), 36-41. doi: 10.1111/cdep.12059

Bull, R., y Scerif, G. (2001). Executive functioning as a predictor of children's mathematics ability: Inhibition, switching, and working memory. Developmental Neuropsychology, 19(3), 273-293.

D'Amico, A., y Passolunghi, M. C. (2009). Naming speed and effortful and automatic inhibition in children with arithmetic learning disabilities. Learning and Individual Differences, 19(2), 170-180.

Davis, A., y Bamford, G. (1995). The effect of imagery on young children's ability to solve simple arithmetic. Education Section Review-British Psychological Society, 19, 61-61.

De Castro, C., Molina, E., Gutiérrez, M. L., Martínez, S., y Escorial, B. (2012). Resolución de problemas para el desarrollo de la competencia matemática en Educación Infantil. Números. Revista de Didáctica de las Matemáticas, 80, 53-70.

Desoete, A., Roeyers, H., y De Clercq, A. (2004). Children with mathematics learning disabilities in Belgium. Journal of Learning Disabilities, 37(1), 50-61.

Diamond, A. (2013). Executive functions. Annual review of psychology, 64, 135-168.

Diamond, A., y Taylor, C. (1996). Development of an aspect of executive control: Development of the abilities to remember what I said and to "Do as I say, not as I do". Developmental Psychobiology, 29(4), 315-334.

Gathercole, S. E., Pickering, S. J., Ambridge, B., y Wearing, H. (2004). The structure of working memory from 4 to 15 years of age. Developmental Psychology, 40(2), 177. doi: 10.1037/00121649.40.2.177

Geary, D. C., Hoard, M. K., Nugent, L., y Byrd-Craven, J. (2008). Development of number line representations in children with mathematical learning disability. Developmental Neuropsychology, 33(3), 277-299.

Grégoire, J., Noël, M. P., y Van Nieuwenhoven, C. (2005). Tedi-Math. Manual. Madrid: TEA.

Henry, L., y MacLean, M. (2003). Relationships between working memory, expressive vocabulary and arithmetical reasoning in children with and without intellectual disabilities. Educational and Child Psychology, 20(3), 5163.

Holmes, J., y Adams, J. W. (2006). Working memory and children's mathematical skills: Implications for mathematical development and mathematics curricula. Educational Psychology, 26(3), 339-366.

IBM Corp. Released (2010). IBM SPSS Statistics for Windows, Version 19.0. Armonk, NY: IBM Corp.

Jordan, N. C., Glutting, J., y Ramineni, C. (2010). The importance of number sense to mathematics achievement in first and third grades. Learning and individual differences, 20(2), 82-88. doi: 10.1016/j.lindif.2009.07.004

Lee, K., Ng, S. F., Bull, R., Pe, M. L., y Ho, R. H. M. (2011). Are patterns important? An investigation of the relationships between proficiencies in patterns, computation, executive functioning, and algebraic word problems. Journal of Educational Psychology, 103(2), 269.

Luria, A. R. (1966). Higher cortical functions in man. New York: Basic Books.

Mercader, J., Presentación, M. J., Siegenthaler, R., Molinero, V., \& Miranda, A. (2017). Motivación y rendimiento académico en matemáticas: un estudio longitudinal en las primeras etapas educativas. Revista de Psicodidáctica, 22(2), 157-163.

McKenzie, B., Bull, R., y Gray, C. (2003). The effects of phonological and visual-spatial interference on children's arithmetical performance. Educational and Child Psychology, 20(3), 93-108

Nguyen, T., Duncan, R. J., y Bailey, D. H. (2019). Theoretical and methodological implications of associations between executive function and mathematics in early childhood. Contemporary Educational Psychology, 58, 276-287.

Passolunghi, M. C., y Lanfranchi, S. (2012). Domain specific and domain general precursors of mathematical achievement: A longitudinal study from kindergarten to first grade. British Journal of Educational Psychology, 82(1), 42-63. 


\section{PSICOLOGÍA, INFANCIA Y EDUCACIÓN}

Peng, P., Namkung, J., Barnes, M., y Sun, C. (2016). A meta-analysis of mathematics and working memory: Moderating effects of working memory domain, type of mathematics skill, and sample characteristics. Journal of Educational Psychology, 108(4), 455. doi: 10.1037/edu0000079

Peng, P., y Fuchs, D. (2016). A meta-analysis of working memory deficits in children with learning difficulties: Is there a difference between verbal domain and numerical domain? Journal of Learning Disabilities, 49(1), 320.

Pickering, S. J., Baqués, J., y Gathercole, S. E. (1999). Bateria de tests de memòria de Treball. Barcelona: Laboratori de Memòria de la Universitat Autònoma de Barcelona.

Siegel, L. S., y Ryan, E. B. (1989). The development of working memory in normally achieving and subtypes of learning disabled children. Child Development, 50, 973-980. doi: 10.2307/1131037

Szucs, D., Devine, A., Soltesz, F., Nobes, A. y Gabriel, F. (2013). Developmental dyscalculia is related to visuospatial memory and inhibition impairment. Cortex, 49, 2674-2688.

Van der Sluis, S., de Jong, P. F., y van der Leij, A. (2004). Inhibition and shifting in children with learning deficits in arithmetic and reading. Journal of Experimental Child Psychology, 87(3), 239-266.

Wang, L. C., Tasi, H. J., y Yang, H. M. (2012). Cognitive inhibition in students with and without dyslexia and dyscalculia. Research in Developmental Disabilities, 33(5), 1453-1461.

Wechsler, D. (1967). Manual for the Wechsler preschool and primary scale of intelligence. New York: Psychological Corporation. 
